\title{
Mathematical Simulation of Remote Detection of Human Breathing and Heartbeat by Multifrequency Radar on the Background of Local Objects Reflections
}

\author{
Alexander S. Bugaev, Institute of Radioengineering and Electronics RAS, Moscow \\ Valeri V. Chapursky, Sergey I. Ivashov, Bauman Moscow State Technical University
}

Key Words: multifrequency radars, human detection, background reflections, mathematical simulation.

\begin{abstract}
SUMMARY
The mathematical description of signal processing basic stages in multifrequency radar intended for detection of human breathing and heartbeat is given. Cross-correlation functions of different kinds of the multifrequency probing signals, modelling optimum processing of impulses are compared in view of resolution, levels of lateral lobes and presence of diffractive maxima. The model of multifrequency radar signal taking into account breath and palpitation of the person is offered. For remote allocation of person's breath and heartbeat realizations with selection on range cells the spectral analysis in each range element is used. Such a selection besides definition of range up to the living human allows excluding reflections from motionless and inactive local objects, available in other range cells.
\end{abstract}

\section{INTRODUCTION}

There is now keen interest to use of microwave radar methods for detection and diagnostics of human beings. The microwave signals reflected from individuals contain biometric information which is related to the periodical reductions of a heart, blood vessels, lungs and other human internals and fluctuations of the skin in the process of breathing and heart beating. These processes are cyclical and the frequency of their recurrence is in the range of $0.8-2.5 \mathrm{~Hz}$ for heart beating and $0.2-0.5 \mathrm{~Hz}$ for breathing. A reflected microwave signal containing biometric information we call as biometric radar signal. The main applications of this method could be:

- detection of alive people who are buried under the building debris as a result of natural disasters, technical calamities or accidents;

- detection of the people and parameters of their movements inside the building in process of law enforcement and antiterrorist operations;

- remote testing of psychological conditions of the persons during the latent or open security checks, for example at the airports (remote lie detector);

- touchless measurement of the heartbeat and breathing of patients, when a contact sensor for some reasons cannot be used.
The useful components of biometric information are preserved in the time domain realization of the radar biometric signal. That realization is nonstationary in general case. Examination of its nonstationarity is needed especially in the case of person psychological condition testing and in medicine. Signal spectrums in frequency domain may be useful in process of alive human detection on the background of local objects reflections and other interferences. The theoretical spectrum estimation of a reflected biometric radar signal on the output of receiver devices of the microwave radar sensor with continuous sine-wave sounding was executed in [1]. The radar with non modulated signal does not allow determining distance up to the person, and also has no selection on range and as a consequence it is subjected to reflections and interferences from whole distance interval. One of possible construction of radar for detection of alive persons with selection on range is application of a wideband or ultra wideband (UWB) multifrequency probing signal. In achieving the range resolution comparable to that of UWB pulse signals, multifrequency signals allow receiving considerably greater average radiation power and detection range.

The primary goal of the given research is mathematical simulating of remote allocation of breath and heartbeat realizations of the person by radar with selection on range cells. Such a selection besides definition of range up to the living person allows excluding reflections from motionless and inactive local objects, available in other range cells. Final stage of radar signal processing must reproduce the time realization of biometric signal. It is supposed, that the model will be useful in studying the opportunity and algorithms of division of breath and heartbeat realizations at subsequent processing a biometric radar signal. Such a division gives the precondition for more reliable allocation of information attributes of person's psychological conditions in stressful situations.

\section{DESCRIPTION OF MULTIFREQUENCY SOUNDING SIGNALS}

We suppose that the radar transmits impulse signal periodically with constant period $T_{r}$ and multifrequency 
impulse duration $\tau_{\text {imp }}$. Consider two radar modes: 1) simultaneous radiation of frequencies or monopulse multifrequency (MMF) signal and 2) consecutive radiation of frequencies or step frequency modulated (SFM) signal [2].

In the first case transmitted MMF impulse represents the sum of $M+1$ frequency components at frequencies $\omega_{m}, m=0,1, \ldots, M$ with common envelope $A(t)$ of an impulse:

$$
s_{T}(t)=\sum_{m=0}^{M} s_{m}(t)=A(t) \cdot \sum_{m=0}^{M} \exp \left(j \omega_{m} t\right),
$$

where simultaneous frequency components are $s_{m}(t)=A(t) \exp \left(j \omega_{m} t\right)$. For example we can define a rectangular envelope, $A(t)=\operatorname{rect}\left(t / \tau_{\text {imp }}\right)$.

In the second case transmitted SFM impulse represents the sum of $M+1$ frequency components spaced in time [2]:

$$
s_{T}(t)=\sum_{m=0}^{M} s_{m}(t)=\sum_{m=0}^{M} A_{0}\left(t-m \tau_{s}\right) \exp \left(j \omega_{m} t\right) .
$$

Here $\tau_{s}=\tau_{\text {imp }} / M$ is a period of frequency steps, $\tau_{s}-\tau_{\max }$ corresponds to duration of rectangular envelope of component pulse, which looks like

$$
A_{0}(t)=\operatorname{rect}\left(t /\left(\tau_{s}-\tau_{\max }\right)\right),
$$

and $\tau_{\max }=2 R_{\max } / c-$ is the time delay corresponding to maximum range $R_{\max }$ for which the radar is designed. Such choice of the period and duration of frequency components of SFM impulse guarantees the absence of time overlapping for reference and received components with different frequencies for working interval of delays.

Usually the case of uniform distribution of frequencies with constant step $\Delta \omega$ is considered:

$$
\omega_{m}=\omega_{0}+(m-1) \cdot \Delta \omega, m=0,1, \ldots, M .
$$

Equivalent envelope duration of MMF impulse is connected to frequency step:

$$
\tau_{\text {imp }}=1 / \Delta f, \Delta f=\Delta \omega /(2 \pi) \text {. }
$$

Equality (5) meets orthogonal property of transmitted MMF components. For rectangular envelope $A(t)$ of unity amplitude when the condition $\Delta f \cdot \tau_{\text {imp }}=1,2,3, \ldots$ holds we have

$$
\int_{-\infty}^{\infty} s_{k}(t) s_{m}^{*}(t) d t=\tau_{i m p} \delta_{k, m}, \quad \text { where } \quad \delta_{k, m}= \begin{cases}1, & k=m \\ 0, & k \neq m\end{cases}
$$

At non integer values of $\Delta f \cdot \tau_{i m p}$ strict orthogonality of the components of rectangular MMF impulse is absent. Also in strict sense components of sounding (reference) MMF impulse and delayed received components reflected from the target cease to be orthogonal.

The other variant of component orthogonality of the MMF impulse is rectangular spectrum components with bandwidth $\Delta \Omega$, smaller or equal to a frequency step $\Delta \omega$, i.e. satisfying the inequality $\Delta \Omega \leq \Delta \omega$.

It is necessary to note, that frequency components of SFM signal are always orthogonal owing to the absence of their overlapping in time domain. For such signals property similar to (6) holds not only for components of the probing signal, but also for different frequencies components of the reference signal and delayed signal frequency components reflected from the target.

\section{CROSS-CORRELATION FUNCTIONS OF MULTIFREQUENCY SIGNALS}

To simulate mathematically the optimum receiver we can use the time domain cross-correlation function of received and reference (matched) multifrequency impulses. Signal crosscorrelation function also determines the potential quality of the radar processing system with a selected sounding signal. This function coincides with section of radar ambiguity function [2] on delay. The width of its main lobe defines radar resolution on range in radar model. The level of side lobes and number of diffraction maxima of this function enable us to simulate suppression of local objects reflections. Thus correlation function or correlation integral enables us to take into account at simulation main characteristics of multifrequency radar: frequency bandwidth $\Delta F$, frequency step $\Delta f$ and number $M+1$ of frequency components. In future investigations the intensity of components, spacing of frequencies with nonuniform step, and also the forming of envelope for MMF and SFM pulses may be subjected to a choice.

For MMF sounding (1) the Received signal with a delay $\tau_{R}$ and the reference signal Matched with a delay $\tau_{M}$ look like

$$
\begin{aligned}
& u_{R}(t)=A\left(t-\tau_{R}\right) \sum_{m=0}^{M} q_{m} \exp \left\{j \omega_{m}\left(t-\tau_{R}\right)\right\} \\
& u_{M}(t)=A\left(t-\tau_{M}\right) \sum_{m=0}^{M} \bar{q}_{m} \exp \left\{j \omega_{m}\left(t-\tau_{M}\right)\right\}
\end{aligned}
$$

where $q_{m}$ and $\bar{q}_{m}$ are propagation factors at frequency $\omega_{m}$.

The correlation integral of signals (7) and (8) is defined by formulas

$$
\begin{gathered}
Q(\Delta \tau)=\int_{-\infty}^{\infty} u_{R}(t) u_{M}^{*}(t) d t= \\
=\sum_{m_{1}=0 m_{2}=0}^{M} \sum_{m_{1}} \bar{q}_{m_{2}} \mathbf{e}^{j \omega_{m_{2}} \Delta \tau} \int_{-\infty}^{\infty} A(t) A(t-\Delta \tau) \mathrm{e}^{j\left(\omega_{m_{1}}-\omega_{m_{2}}\right) t} d t= \\
=2 \pi \sum_{m_{1}=0}^{M} \sum_{m_{2}=0}^{M} q_{m_{1}} \bar{q}_{m_{2}} \mathbf{e}^{j \omega_{m_{2}} \Delta \tau} \int_{-\infty}^{\infty} G\left(\omega-\omega_{m_{1}}+\omega_{m_{2}}\right) G(\omega) \mathbf{e}^{j \omega \cdot \Delta \tau} d t
\end{gathered}
$$

Here $\Delta \tau=\tau_{M}-\tau_{R}$, and $G(\omega)$ is a spectrum of $A(t)$ :

$$
G(\omega)=\frac{1}{2 \pi} \int_{-\infty}^{\infty} A(t) \mathrm{e}^{-j \omega t} d t
$$

Further for simplicity we shall assume alignment of multipliers $q_{m}$ and $\bar{q}_{m}$, suppose they are independent from $m$ and equal to unit.

$$
\begin{gathered}
\text { At a rectangular envelope of MMF pulse we have: } \\
\qquad Q(\Delta \tau)=[b(\Delta \tau)-a(\Delta \tau)] \cdot h[b(\Delta \tau)-a(\Delta \tau)] \times \\
\sum_{m_{1}, m_{2}=0}^{M} \operatorname{sinc}\left[\left(\omega_{m_{1}}-\omega_{m_{2}}\right) \frac{b(\Delta \tau)-a(\Delta \tau)}{2 \pi}\right] \mathbf{e}^{j \omega_{m_{2}} \Delta \tau+j\left(\omega_{m_{1}}-\omega_{m_{2}}\right)(b(\Delta \tau)+a(\Delta \tau)) / 2}
\end{gathered}
$$


where $\quad a(\Delta \tau)=\max (0, \Delta \tau), \quad b(\Delta \tau)=\min \left(\tau_{i m p}, \tau_{i m p}+\Delta \tau\right)$, $h(x)$ is Heaviside function or unit step function, $\operatorname{sinc}(y)=\sin (\pi y) /(\pi y)$.

Under the condition $\tau_{i m p}>\Delta f^{-1}$ expression (10) can be submitted as the single sum:

$$
Q_{1}(\Delta \tau)=[b(\Delta \tau)-a(\Delta \tau)] \cdot h[b(\Delta \tau)-a(\Delta \tau)] \sum_{m=0}^{M} \mathbf{e}^{j \omega_{m} \Delta \tau}
$$

Under the given condition components of different frequencies become almost orthogonal and their cross correlation tends to zero. With additional condition $|\Delta \tau| \leq \Delta f^{-1}$ it is possible to neglect first two factors in (11):

$$
Q_{2}(\Delta \tau)=\sum_{m=0}^{M} \mathbf{e}^{j \omega_{m} \Delta \tau}
$$

At a rectangular spectrum of $M M F$ pulse with frequency components bandwidth $\Delta \Omega, \quad$ i.e. at $G(\omega)=\operatorname{rect}[(\omega+\Delta \Omega / 2) / \Delta \Omega]$, and under the condition $\Delta \omega \geq \Delta \Omega$ for correlation integral we have the expression:

$$
Q(\Delta \tau)=2 \pi \Delta \Omega \operatorname{sinc}\left[\frac{\Delta \Omega \cdot \Delta \tau}{2 \pi}\right] \sum_{m=0}^{M} \mathbf{e}^{j \omega_{m} \Delta \tau} .
$$

For SFM sounding (2) received and reference signals are as follows:

$$
\begin{aligned}
& u_{R}(t)=\sum_{m=0}^{M} q_{m} A_{0}\left(t-\tau_{R}-m \tau_{s}\right) \mathbf{e}^{j \omega_{m}\left(t-\tau_{R}\right)}, \\
& u_{M}(t)=\sum_{m=0}^{M} A_{1}\left(t-m \tau_{s}\right) \mathbf{e}^{j \omega_{m}\left(t-\tau_{M}\right)},
\end{aligned}
$$

where $A_{1}(t)=\operatorname{rect}\left(t / \tau_{s}\right)$.

Under the conditions $\tau_{M}<\tau_{\max }$ and $\tau_{R}<\tau_{\max }$ the expression for correlation integral has a view of the unitary sum in view of full orthogonality of different frequency pulses in time domain, as against a case of the MMF signal. As well as earlier, accepting $q_{m}=$ const, we receive:

$$
\begin{aligned}
Q(\Delta \tau) & =\sum_{m=0}^{M} \int_{-\infty}^{\infty} A_{0}\left(t-\tau_{R}-m \tau_{s}\right) A_{1}\left(t-m \tau_{s}\right) d t \cdot \mathbf{e}^{j \omega_{m}\left(\tau_{M}-\tau_{R}\right)}= \\
& =\left(\tau_{s}-\tau_{\text {max }}\right) \sum_{m=0}^{M} \mathbf{e}^{j m \Delta \tau} .
\end{aligned}
$$

The given exact formula with the constant omitted coincides with formula (12) which represents approximate expression of correlation integral in the case of the MMF signal at a big impulse duration.

Calculations by formulas (10)-(13) and (16) were carried out at the following signal parameters: mean frequency of initial frequency component $f_{0}=2 \mathrm{GHz}$, total bandwidth $\Delta F=300 \mathrm{MHz}$, number of frequency components $M+1=32$. On Fig. $1-3$ the normalized dependences of correlation integral modulus for MMF pulse, designed for a case $\tau_{i m p}=\Delta f^{-1}$ under the exact formula (10) and with the help of the approximate expressions (11) - (12) are submitted. The dashed line represents correlation function of rectangular pulse envelope. On Fig. 1 and 2 interval of delays $\Delta \tau$ on a horizontal axis corresponds to impulse duration,
$|\Delta \tau| \leq \tau_{\text {imp }}=\Delta f^{-1}$, where $\Delta f=\Delta F / M$. On Fig. 3 the same dependences are shown on the smaller interval $|\Delta \tau| \leq 2 \Delta F^{-1}$ appropriate to double resolution on delay. Essential difference of the exact and approximate results is visible, especially in the field of the main lobe and diffractive lobes. Calculations have shown, that the given differences become practically not essential at increase of impulse duration in some times, for example in the case when $\tau_{\text {imp }}=3 \Delta f^{-1}$.

Having noted that function $Q_{2}(\Delta \tau)$ actually coincides with the exact expression of correlation integral for SFM signal (16), the following conclusion is fair. Under the condition of frequency components orthogonality $\tau_{\text {imp }}=\Delta f^{-1}$ resolution of MMF impulse with rectangular envelope surpasses resolution of SFM signal approximately one and a half times. Due to rectangular envelope of MMF pulse full suppression of distant diffractive lobes, which are typical for SFM signal, takes place. However full suppression of two nearest diffractive lobes does not occur, and their level amounts $40 \%$ from the main lobe level.

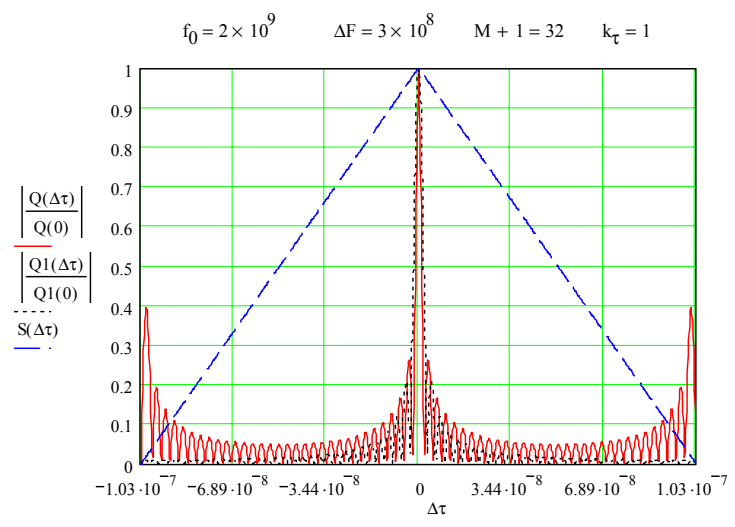

Fig.1. Cross-correlation function of MMF rectangular pulse at $\tau_{\text {imp }}=\Delta f^{-1}$, calculated on the interval $|\Delta \tau| \leq \Delta f^{-1}$ by exact (continuous line) and approximate (dot line) formulas (10) and (11).

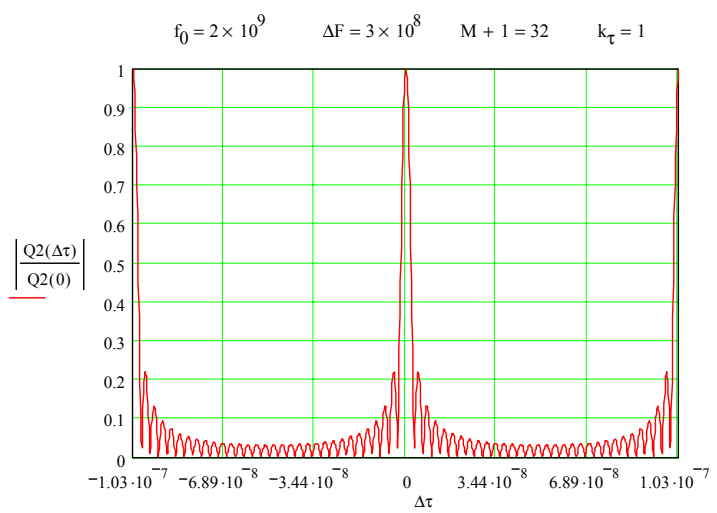

Fig.2. Cross-correlation function of MMF rectangular pulse at $\tau_{\text {imp }}>\Delta f^{-1}$, calculated on the interval $|\Delta \tau| \leq \Delta f^{-1}$ and coincided with dependence for SFM a signal. 


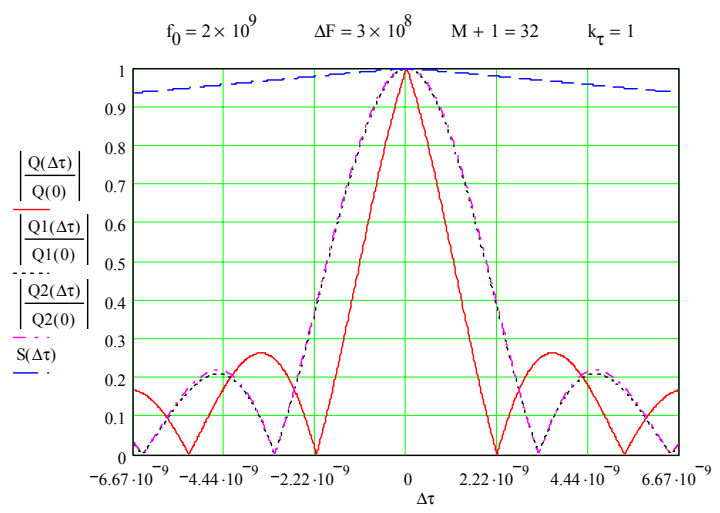

Fig.3. Cross-correlation function of MMF rectangular pulse at $\tau_{i m p}=\Delta f^{-1}$, calculated on the interval $|\Delta \tau| \leq 2 \Delta F^{-1}$ by exact (continuous line) and approximate formulas (10), (11) and (12).

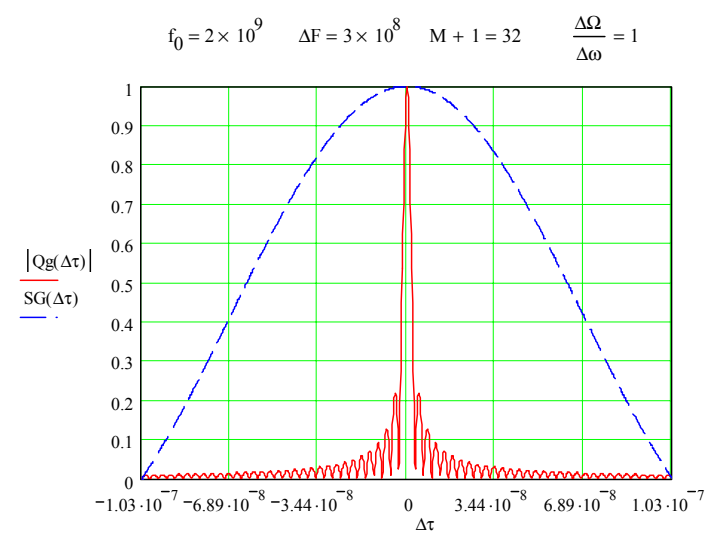

Fig.4. Cross-correlation function of MMF pulse with a rectangular spectrum of frequency components at $\Delta \omega=\Delta \Omega$, calculated on the interval $|\Delta \tau| \leq \Delta f^{-1}$.

Other results take place for MMF impulse with a rectangular spectrum of frequency components. Diagrams on Fig 4 are designed by the exact formula (16) which is equitable in bandwidth $\Delta \Omega$ of the frequency components equal to a frequency step $\Delta \omega$. The dashed line meets to pulse envelope. In this case both distant and near diffractive lobes are completely suppressed. At the same time number of frequency components can be reduced without occurrence of ambiguous measurements and diffractive lobes.

\section{SIMULATION OF BIOMETRIC SIGNALS AND THEIR PROCESSING IN MULTIFREQUENCY RADAR.}

First we describe mathematical model of the multi frequency biometric radar signal reflected from the set of targets, which includes living persons with breath and heartbeat and motionless local objects. For the convenience of simulating unified numbering of persons and motionless objects is entered according to their serial numbers $n$, $0 \leq n \leq N$. The model takes into account the body skin vibrations caused by breath and heartbeat. In general case the law of reflected signal delay modulation of a received multi frequency signal of the $n$-th person represents the sum of quasi-periodic sine waveform with varying breath rate period $T_{b}(t, n)$ and sequence of heartbeat impulses with varying period $T_{h}(t, n)$. For example, if the period of breath is constant, and the period of heartbeat changes periodically under the sine law, then we have

$$
T_{b}(t, n)=T_{b n}^{0} \text { and } T_{h}(t, n)=T_{h n}^{0}\left(1+m_{n} \cdot \sin \left(2 \pi F_{h n}\right)\right),
$$

where $T_{b n}^{0}, F_{h n}$ and $m_{n}<<1$ are approximation parameters for person with number $n$. Deviations of signal delay modulation are proportional to displacements $\Delta_{b n}$ и $\Delta_{h n}$ of the $n$-th body surface at breath and heartbeat. Then simple approximation of delay variations and corresponding phase variations on the $i$-th frequency of Received multifrequency signal of the $n$-th person can be given as follows

$$
\begin{aligned}
\tau_{R}(t, n)= & \tau_{0 n}+\Delta \tau(t, n)= \\
\frac{2}{c}\left[r_{0 n}+\right. & \left.\Delta_{b n} \sin \left(\frac{2 \pi t}{T_{b}(t, n)}+\varphi_{b n}\right)+\Delta_{h n} \sin ^{2 q}\left(\frac{2 \pi t}{T_{h}(t, n)}+\varphi_{h n}\right)\right] \\
& \varphi_{m}(t, n)=-\varphi_{0 n m}-\Delta \varphi_{m}(t, n)+\psi_{n}= \\
= & -\frac{2 \omega_{m} r_{0 n}}{c}-\omega_{m} \cdot \Delta \tau(t, n)+\psi_{n}
\end{aligned}
$$

There are some optional designations: $c$ - is the speed of electromagnetic waves propagation, $q>>1-$ is an integer parameter of heart pulse approximation, $\omega_{m}-$ the $m$-th radiated frequency, $r_{0 n}$ and $\psi_{n}$ - average distance and constant phase of the $n$-th target. If number $n$ belongs to a motionless local object then

$$
\begin{gathered}
\tau_{R}(t, n)=\tau_{0 n}=\frac{2 r_{0 n}}{c}, \\
\varphi_{m}(t, n)=-\varphi_{0 n m}+\psi_{n}=-\frac{2 \omega_{m} r_{0 n}}{c}+\psi_{n} .
\end{gathered}
$$

The example of received signal phase modulation functions for two persons (number 1 and 3 ) with differing frequencies of breath $(0.2 \mathrm{~Hz}$ and $0.3 \mathrm{~Hz})$ and the heartbeat $(1.5 \mathrm{~Hz}$ and $2.0 \mathrm{~Hz})$, calculated by (18)-(19) for six different frequencies $(m=0,1, \ldots, 5)$ in $300 \mathrm{MHz}$ bandwidth near $2 \mathrm{GHz}$ with uniform step of $60 \mathrm{MHz}$, is submitted on Fig. 5. Accepted body surface displacements due to breath and heartbeat were $\Delta_{b n}=0.5 \mathrm{~cm}$ and $\Delta_{h n}=0.5 \mathrm{~mm}$.

If we suppose that the radar transmits MMF pulses periodically with constant period $T_{r}$, then the $m$-th frequency component of received multi frequency signal reflected from $N+1$ targets in the $k$ - th sounding period can be represented as follows:

$$
s_{m k}^{R}(t)=\sum_{n=0}^{N} \dot{U}_{n} A\left(t-\tau_{R}\left(k T_{r}, n\right)-k T_{r}\right) \exp \left[j \omega_{m}\left(t-\tau_{R}\left(k T_{r}, n\right)\right)\right],
$$

The whole received MMF impulse in the $k$ - th sounding period is

$$
u_{R}(t, k)=\sum_{m=0}^{M} q_{m} s_{m k}^{R}(t) .
$$


In (21) complex value $\dot{U}_{n}=U_{n} \exp \left(j \Psi_{n}\right)$ represents the signal reflection amplitude of the $n$ - th target and $A(t)$ is a pulse envelope

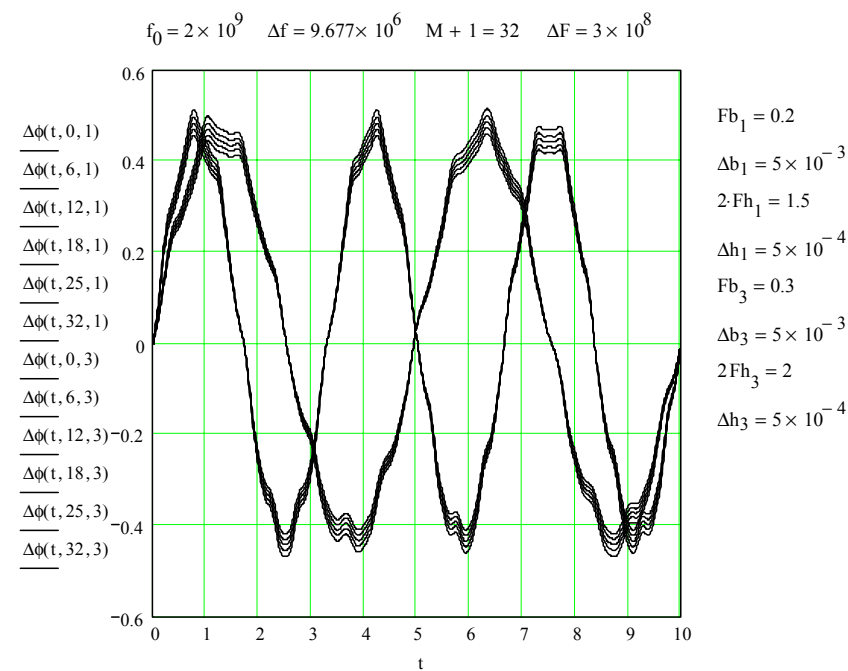

Fig. 5. Phase increment dependences of biometric signals for two persons with different breath and heartbeat rates on six frequencies.

function. We assume constant phases and reflection coefficients independent from frequencies in frequency bandwidth $\Delta F$. As above we moreover assume alignment of multipliers $q_{m}$ and their equality to unit.

Biometric signal processing in the multifrequency radar includes the multi channel correlation of received multifrequency impulse in the $k$-th sounding period with reference impulse, corresponding to some Matched constant delay $\tau_{M}$ :

$$
u_{M}(t, k)=A\left(t-\tau_{M}-k T_{r}\right) \sum_{m=0}^{M} \exp \left\{j \omega_{m}\left(t-\tau_{M}\right)\right\} .
$$

After substitution of (22) and (23) correlation integral for the $k$-th sounding period can be represented as follows:

$$
Q_{\Sigma}\left(k, \tau_{M}\right)=\int_{-\infty}^{\infty} u_{R}(t, k) u_{M}^{*}(t, k) d t=\sum_{n=0}^{N} \dot{U}_{n} Q\left(\tau_{R}\left(k T_{r}, n\right)-\tau_{M}\right),
$$

where $Q(\Delta \tau)$ represents usual correlation integral (10) of MMF impulse from the previous section or one of its approximate form (11)-(13). Under the condition $M \tau_{s}<<T_{r}$ one can obtain the result, completely analogous to (24) also for SFM signal. In that case one must use correlation integral $Q(\Delta \tau)$ in the form (16).

For MMF signal with corresponding pulse duration $\tau_{i m p} \gg \Delta f^{-1}$ or for SFM signal with the same parameters as on Fig.6 example of delay distribution of correlation integral modulus for six targets $(N+1=6)$, uniformly arranged on distance $\left(r_{0 n}=2,3.2,4.4,5.6,6.8,8.8 \mathrm{~m}\right)$ is presented on Fig.6. Targets with numbers $n=1,3$ (from left to right, beginning with $n=0$ ) are living persons, and targets with numbers $n=0,2,4,5$ are motionless local objects. Radar cross sections (RCS) for local objects by convention are accepted $10 \mathrm{~m}^{2}$, but for human bodies it consists $1 \mathrm{~m}^{2}$.

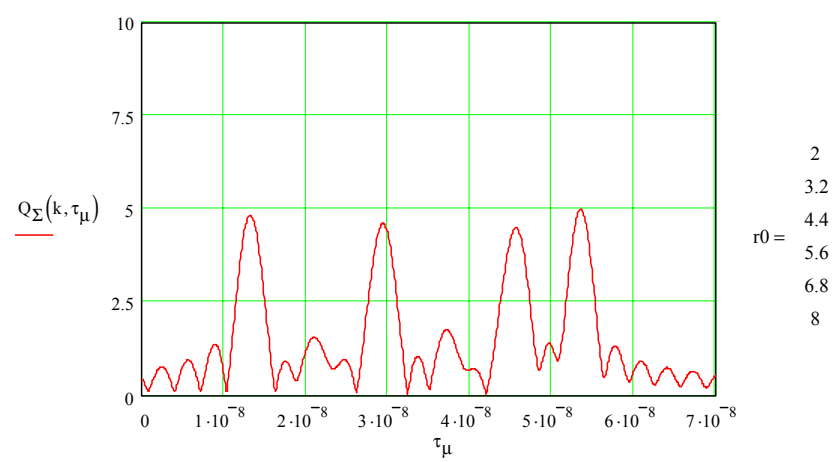

Fig. 6. Example of delay distribution of correlation integral modulus for six targets.

For detection of alive person on breath and heartbeat the correlation processing is made for all expected range cells with the period $T_{r}$ answering to the sampling theorem at the upper frequency of heartbeat spectrum. Samples readout is carried out in discrete time moments $k T_{r}$ on the great time interval of tens seconds to obtain the sufficient frequency resolution. Then FFT representation of correlation integral modulus is made in a range-frequency plane:

$$
G\left(\Omega_{m}, \tau_{\mu}\right)=\left|F F T_{m}\left\{Z\left(k, \tau_{\mu}\right)\right\}\right| .
$$

Examples of 2D topographic distributions of $(7)$ in $(\Omega, \tau)$ plane are displayed on Fig.7 and Fig. 8. On Fig.9 topographic distribution is the same as on Fig. 8 but in logarithmic scale.

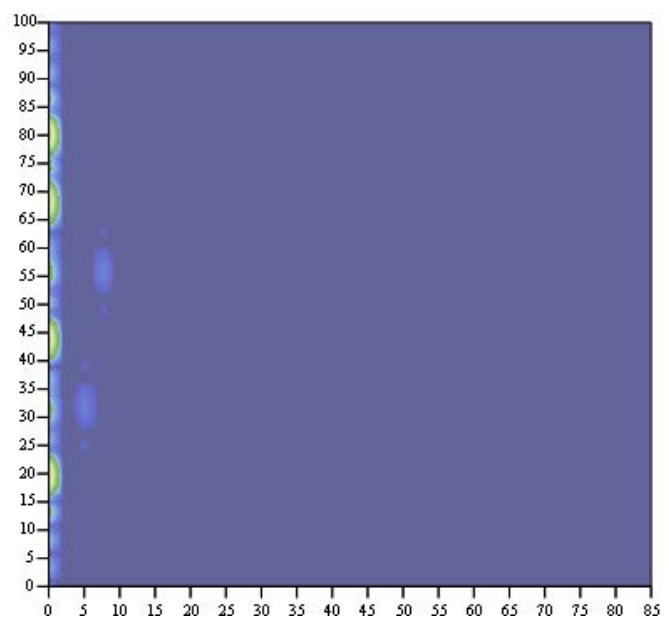

$\mathrm{m} 0$

Fig. 7. Topographic distributions of output modulus of signal processing system for two different persons and four local objects on conditions of Fig.5,6.

In Fig. 7 the parameter proportional to range is postponed on the vertical axis, and the parameter equal to frequency sample number of FFT is postponed on the horizontal axis. Fig.8 differs from Fig.7 by suppression the near zero frequency components on which motionless local objects are disposed. Suppression near zero frequency spectrums rejects 
local objects and improves the visibility of heartbeat components near frequency samples with numbers $m=38$ and $m=52$ for living person's numbers $n=1$ and $n=3$ at distances $3.2 \mathrm{~m}$ and $5.6 \mathrm{~m}$. Maxima near frequency samples with numbers $m=5$ and $m=7-8$ correspond to breathing rates of the same persons.

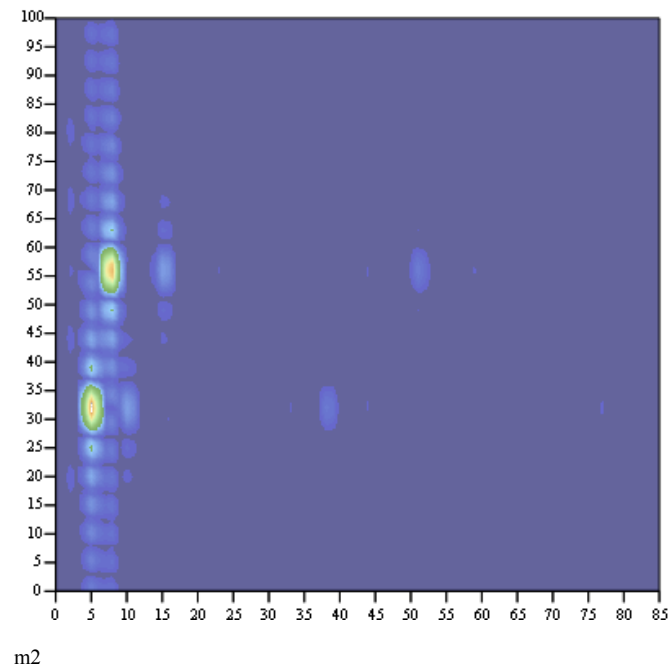

Fig. 8. Topographic distributions of output modulus of biometric radar's signal processing system with application of local objects rejector for two different persons and four local objects on conditions of Fig. 7.

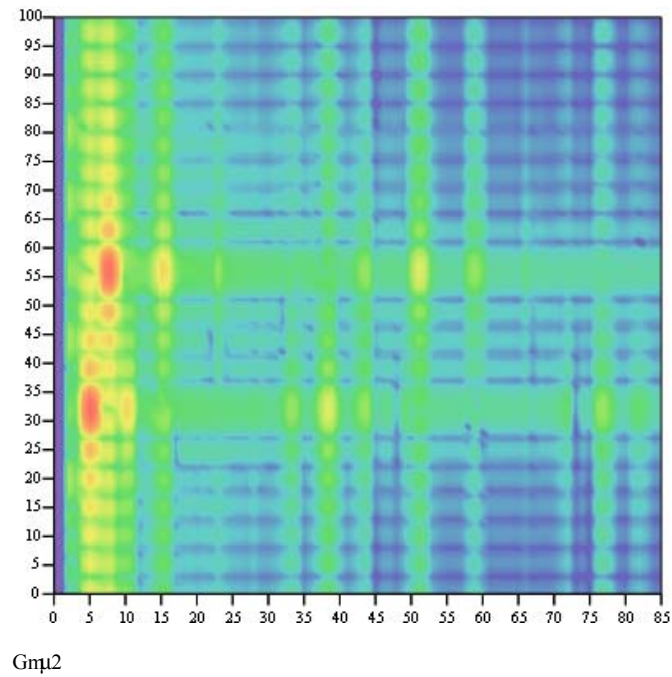

Fig. 9. The same as on Fig. 8 represented in logarithmic scale.

On topographical diagrams the second harmonic of breath is visible well. In logarithmic scale on Fig. 9 the supreme and combinational harmonics of frequencies of breathing and heartbeat are visible also. All these components are caused by process of phase modulation of a sounding signal due to vibrations of human's skin and internal at breathing and heartbeat. The analysis of the data in the displayed planes allows allocating the range cell in which there are living persons and determining their distances. Focusing of signal processing algorithm on this range cells allows reproducing time realizations of breath and heartbeat of detected persons.
The given restoration occurs almost in the initial kind similar to either of realizations among the submitted on Fig. 5 and corresponding to one of frequencies.

\section{CONCLUSIONS}

As applied to detection of alive people on breathing and heartbeat the mathematical model of a multifrequency radar sounding was examined. The analysis of correlation integral for monopulse multifrequency signal and signal with step frequency modulation has shown the presence of higher delay resolution in the first case. Comparison of simulated outputs of biometric radar signal processing system for two persons and four local objects on different distances without rejecting of local object signals and with application of local objects rejector revealed possibility of persons detection. Also reproducing of a total biometric signal of breathing and heartbeat is possible from that range cell in which the person is located.

\section{ACKNOWLEDGEMENTS}

Support for this work was provided by the International Science and Technology Center and the Russian Foundation for Basic Research.

\section{REFERENCES}

1. A.S. Bugaev, V.V. Chapursky, S.I. Ivashov, V.V. Razevig, A.P. Sheyko, I.A. Vasilyev. Through wall sensing of human breathing and heart beating by Monochromatic Radar. Proceedings of the Tenth International Conference on Ground Penetrating Radar. June 21-24, 2004, V.1, pp. 291-294. Delft, the Netherlands.

2. D. R. Wehner. High Resolution Radar. The Artech House, 1987.

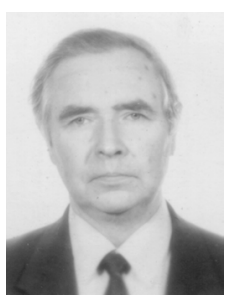

Valery V. Chapursky was born in Vologda, Russia in 1942. He graduated from Moscow Power Engineering Institute on Radio physics and Electronics in 1965. He took Candidate of Technical Sciences and Doctor of Technical Sciences degrees in Radar Technology in 1971 and 1997 respectively. Many years he was with the Central Scientific and Research Institute of Radio Electronic Systems (TsNIIRES). Now he is main scientist of R\&D Institute of Applied Mathematics and Mechanics at Bauman Moscow State Technical University. His main research interests include radio holography, UWB and GPR radars, image recognition according to neural nets algorithms. Dr. Capursky is a member of IEEE and he is awarded by a title of "Honorary Radio specialist of Russia" in 1996.

R\&D Institute of Applied Mathematics and Mechanics at the Bauman Moscow State Technical University. $5,2^{\text {nd }}$ Bauman str., Moscow, 105005, Russia.

Tel/fax: +7 (095) 632-22-19

E-mail : rslab@,rslab.ru, vchapursky@,rslab.ru 